\section{S93 EFFECT OF LUMACAFTOR/IVACAFTOR ON TOTAL, BRONCHIECTASIS, AND AIR TRAPPING COMPUTED TOMOGRAPHY (CT) SCORES IN CHILDREN HOMOZYGOUS FOR F508DEL-CFTR: EXPLORATORY IMAGING SUBSTUDY}

${ }^{1}$ AS Brody, ${ }^{2} \mathrm{~S}$ Nagle, ${ }^{3} \mathrm{C}$ Hug, ${ }^{3} \mathrm{G}$ Marigowda, ${ }^{3} \mathrm{D}$ Waltz, ${ }^{4} \mathrm{~J}$ Goldin, ${ }^{5} \mathrm{~F}$ Ratjen, ${ }^{3} \mathrm{~L}$ Wang. ${ }^{1}$ Cincinnati Children's Hospital Medical Centre, Cincinnati, US; ${ }^{2}$ University of Wisconsin, Madison, US; ${ }^{3}$ Vertex Pharmaceuticals Incorporated, Boston, US; ${ }^{4}$ David Geffen School of Medicine, Los Angeles, US; ${ }^{5}$ University of Toronto, Toronto, Canada

\subsection{6/thoraxjnl-2017-210983.99}

Objective To evaluate the effects of lumacaftor/ivacaftor (LUM/ IVA) combination therapy on lung morphology and physiology with computed tomography (CT) scanning in patients aged 6 to 11 years with cystic fibrosis (CF) homozygous for the F508del-CFTR mutation.

Methods Baseline CT scans were obtained in 19 patients (12 active treatment, 7 placebo) from the phase 3 LUM/IVA trial (NCT02514473), and 24 week CT scans were completed in 7 active treatment and 3 placebo patients. CT scans were obtained at total lung capacity and at residual volume (RV). CT scans were scored by 2 independent readers blinded to all patient and time point information using the Brody score, which evaluates the extent and severity of multiple aspects of CF lung disease, including bronchiectasis and air trapping. Scores are presented as mean (SD); no statistical testing was performed for this preliminary study.

Results Mean total CT score (sum of the subcomponent scores) decreased from 20.6 to 12.5 (mean change [SD], 8.1 [13.6]) in the LUM/IVA group and increased from 32.8 to 41.4 (8.6 [14.6]) in the placebo group. The mean bronchiectasis score decreased from 3.2 to $2.5(0.7$ [1.3]) in the LUM/ IVA group and increased from 6.4 to 8.1 (1.7 [2.1]) in the placebo group. Additionally, the diameter of ectatic bronchi decreased in several patients on active treatment. The mean air trapping score decreased from 7.8 to 5.9 (mean difference [SD], 1.9 [6.8]) in the active group and increased from 9.8 to 14.5 (4.7 [11.7]) in the placebo group, as shown for 1 patient (figure 1).

Conclusion This is the first report to describe CT lung findings after CFTR corrector/potentiator therapy in patients aged 6 to 11 years homozygous for F508del. In this 24 week exploratory analysis, bronchiectasis and air trapping scores improved in patients treated with LUM/IVA and worsened in the placebo group. These data suggest that LUM/IVA treatment may reduce CF disease-related changes in lung morphology and/or physiology and support the need for further study.

Please refer to page A257 for declarations of interest in relation to abstract $\mathrm{S} 93$.
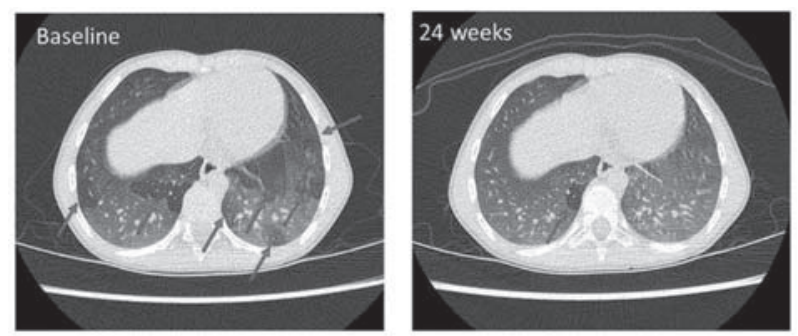

Abstract S93 Figure 1 Computed tomography images (residual volume) showing air trapping (arrows) at baseline and 24 weeks in a patient treated with lumacaftor/ivacaftor.

\section{S94 \\ DEVELOPMENT OF ASSAYS TO ASSESS SAFETY AND EFFICACY OF LENTIVIRAL GENE THERAPY FOR CYSTIC FIBROSIS}

${ }^{1} \mathrm{AD}$ Saleh, ${ }^{1} \mathrm{NK}$ Clarke, ${ }^{1} \mathrm{C}$ Meng, ${ }^{2} \mathrm{MR}$ Jacobson, ${ }^{1} \mathrm{C} \mathrm{C}$ Davies, ${ }^{2}$ SR Durham, ${ }^{1}$ EWFW Alton, ${ }^{1} \mathrm{U}$ Griesenbach. 'Department of Gene Therapy, NHLI, Imperial College and The UK CF Gene Therapy Consortium, London, Oxford, Edinburgh, UK; ${ }^{2}$ Allergy and Clinical Immunology, Inflammation Repair and Development, NHLI, Imperial College, London, UK

\subsection{6/thoraxjnl-2017-210983.100}

Introduction The UK Cystic Fibrosis Gene Therapy Consortium has developed a novel lentiviral vector (rSIV.F/HN) designed to transduce airway epithelial cells efficiently and is preparing a first-in-man lentiviral gene therapy trial building on the recent success of the repeat-dosing non-viral trial. Assays to determine safety and efficacy of lentivirus-mediated gene transfer are now being evaluated. Here, we report validation of two comparatively novel techniques.

Methods and Results Digital droplet (DD) RT-PCR: Virus particles may be shed over time following topical administration to the airways. DD-RT-PCR allows absolute and sensitive quantification of vector genomes. Saliva and urine samples were spiked with known quantities of vector particles (VP) to determine recovery and lower limit of detection (LLD) of the assay. Recovery rates in both body fluids were dependent on the amount of input RNA (Saliva: 7.1\%-15.7\% when spiked with 20-400 VP/uL, urine: $42.5 \%-76 \%$ when spiked with 20 $400 \mathrm{VP} / \mathrm{uL}$ ). The LLD is 200 and $400 \mathrm{VP} / \mathrm{ml}$ in urine and saliva, respectively. However, in an average batch only approximately $1: 700$ to $1: 1000 \mathrm{VP}$ is able to transduce a cell.

In situ hybridisation Quantification of the number of airway cells transduced after pulmonary gene transfer has, to date, not been feasible. In situ hybridisation has traditionally suffered from poor sensitivity and high signal-to-noise ratio. RNAScope (ACDBio) is a novel in situ hybridisation technique based on overlapping probes and multiple levels of signal amplification to enhance specificity and sensitivity, respectively. A549 cells were transduced with $\mathrm{rSIV.F} / \mathrm{HN}$ or were left untransduced as controls. Cells were harvested 4 hours after gene transfer to assess whether RNAScope (using a lentiviral vector-specific sequence) was able to detect vector genomes in transduced cells. Vector genomes were detectable in transduced, but not untransduced cells (figure) and we are currently evaluating the technique in lungs of mice and sheep models.

Conclusion Both DD-RT-PCR and RNAScope hold promise for assessment of safety and efficacy in the upcoming lentivirus CF gene therapy trial.
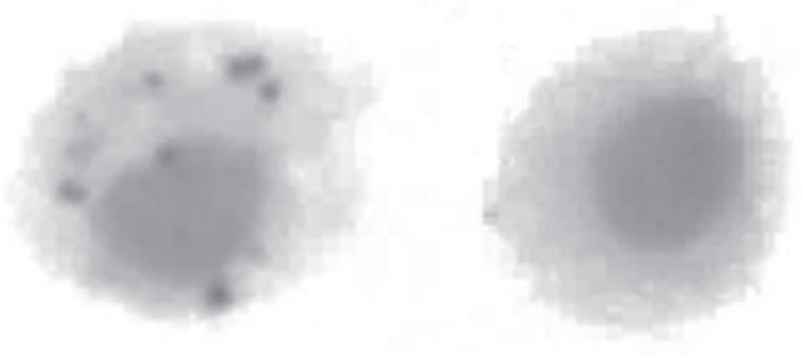

Abstract S94 Figure 1 Left: transduced A549 cell, right: untransduced A549 cell. Vector particles were visualised using RNAScope (red signal). 